\title{
Dynamic Bandwidth Allocation with Optimal Wavelength Switching in TWDM-PONs
}

\author{
Abhishek Dixit, Bart Lannoo, Didier Colle, Mario Pickavet, and Piet Demeester \\ Dep. of Information Technology, Ghent University - iMinds, Gaston Crommenlaan 8/201, B-9050 Gent, \\ Belgium \\ Tel: +32 933149 77, Fax: +32933148 99, e-mail: abhishek.dixit@intec.ugent.be
}

\begin{abstract}
Time and wavelength division multiplexed passive optical networks (TWDM-PONs) have been widely considered as one of the next evolutionary steps of optical access networks. A variety of algorithms exists that explore the problem of scheduling and wavelength assignment in TWDM-PONs. These algorithms, however, allow unlimited switching of wavelengths. In reality, wavelength switching increases guard bands due to the tuning and the switching time of components, limiting channel utilization and increasing packet delays. We propose a novel dynamic bandwidth allocation (DBA) algorithm for TWDM-PON that minimizes the performance degradation due to excessive wavelength switching.
\end{abstract}

Keywords: Dynamic Bandwidth Allocation, Passive Optical Networks, and TDMA/WDMA PON.

\section{INTRODUCTION}

The continuous growth of bandwidth intensive applications like high-definition internet video, file sharing and video conferencing requires a concurrent growth in bandwidth capacities, which can be achieved by bringing an optical fibre closer to an end user, leading to the fibre-to-the-home (FTTH) technology. A promising and widely adopted FTTH technology is a passive optical network (PON). The PON has a tree topology and consists of an optical line terminal (OLT) in a central office, passive splitters/combiners in a remote node (RN) and a number of optical network units (ONU) at a user side. The PON technology can be implemented in a variety of ways, and time and wavelength division multiplexed PON (TWDM-PON) has been adopted by FSAN as the primary candidate of the next generation PON solution. TWDM-PON combines the expansion in capacity deposited by WDM with the inherent resource granularity of a TDM PON. TWDM-PON requires a dynamic bandwidth allocation (DBA) algorithm that dynamically assigns wavelengths and time slots to the ONUs. These DBAs are also known as upstream scheduling and wavelength assignment (USWA) algorithms.

TWDM-PON is realized with a tuneable laser in each ONU. There exist several types of tuneable lasers: the slowest can switch between wavelengths in times ranging from several seconds to a few microseconds, whereas the fastest can switch in nanoseconds. Unfortunately, fast tuneable lasers are very costly and energy consuming, and thus would not be available for access networks. Thus, most often the ONUs will be equipped with lasers having long tuning times (at least tens of microseconds) and the DBA algorithms must take into account the performance degradation inherent to the long tuning and switching times. In addition, completely static allocation of wavelengths is also inefficient. This entails designing a DBA algorithm that switches wavelengths optimally in accord with the laser tuning times.

Current state-of-the-art DBA algorithms, like earliest finish last transmission time (EFT), do not take into account the tuning and the switching time of lasers (and receivers), leading to a very high wavelength switching at ONUs. A very high switching leads to a high switching latency, which degrades the DBA performance. From extensive simulations, we show the performance of EFT degrades with an increase in tuning times: channel utilisation drops to less than $55 \%$ and packet delay increases more than $10 \mathrm{~ms}$ for a tuning time of $100 \mu \mathrm{s}$.

In this paper, we investigate the optimal ONU switching based on the switching and the tuning time considerations, and propose the earliest finish last transmission time - optimal switching (EFT-OS) algorithm which extends the EFT algorithm to switch wavelengths optimally considering the switching and tuning time of laser. EFT-OS adapts the wavelength switching to the laser tuning times, and thus obtains high performance.

The remainder of the paper is organized as follows. Section 2 gives the research overview. Section 3 presents our proposed EFT-OS algorithm. Then, we discuss simulation results (Section 4) and conclusions (Section 5).

\section{RESEARCH OVERVIEW}

USWA can be approached by using either separate time and wavelength scheduling (STWS) algorithms or joint time and wavelength scheduling (JTWS) algorithms [1] (Fig. 1). STWS algorithms decouple the wavelength assignment from time slot allocation and are thus less complex. JTWS algorithms are more complex but an efficient and scalable approach of USWA.

STWA algorithms present simple solutions of USWA. The wavelength assignment can either remain static or vary with load. The static wavelength assignment, however, is not able to obtain goods of statistical multiplexing. Hence, a periodic load dependent grouping of ONUs on wavelengths has been proposed in [2] to 
adapt to bursty traffic. The number of wavelengths can also be changed according to the load to procure energy efficiency. Hybrid sleep mode aware (HSMA) algorithm is proposed to attain significant energy efficiency in TWDM-PONs [2].

In JTWS algorithms, the problem of wavelength assignment is tackled by using the first available wavelength (FAW) [3]. The channel availability is decided based on a "horizon approach" in which each channel is considered as busy until the end of the last scheduled reservation. If no channel is free, then the wavelength with the earliest finishing last transmission (EFT) is selected and the grant for the new reservation is delayed accordingly. Another variation of FAW is latest finish time (LFT). Here the selected wavelength must have the latest finish time among all channels. In horizon-based approaches, the reservation of channels can also be delayed to some extent to have a better scheduling, ultimately leading to reduced average delays [4]. The approach is called as detaining latest finish time (DLFT). The horizon-based approaches, however, create voids and reduce channel utilization.

To improve the low channel utilization of horizon-based algorithms, the void filling approach has been proposed [3]. The OLT tracks voids (unused transmission slot) in all upstream wavelengths and schedules the transmission for an ONU within the first void that is greater than the granted upstream data and the accompanying report message. Similar to horizon-based approaches, here again the transmission can be scheduled on either the earliest void or latest void giving rise to EFT-VF or LFT-VF algorithm. The complexity of these algorithms is higher and is dependent on the number of voids. In contrary, void filling algorithms achieve a better delay performance because of exploiting better upstream scheduling opportunities. The efficiency gap was shown to become more significant with increasing split ratios and differential distances among ONUs, and thus a significant fraction of voids was further minimised by using distance-based grouping (DBG) [5]. DBG maps ONUs with similar round-trip times to the same set of upstream wavelengths, minimizing the differential round-trip delays within each group, leading to significantly shorter voids, higher channel utilization and consequently lower packet delays. Note that DBG is STWS algorithm as wavelength allocation is based on distances of the ONUs rather than load.

However, the JTWS algorithms allow unlimited wavelength switching; as the tuning time of the ONUs is nonnegligible, these algorithms do not achieve acceptable performance even for tuning times in the order of microseconds. Thus, the wavelength assignment algorithm must consider wavelength switching as an important design criterion. This paper proposes such a DBA paradigm that leads to optimal wavelength switching and can be extended over EFT/LFT or even EFT-VF/LFT-VF algorithms.

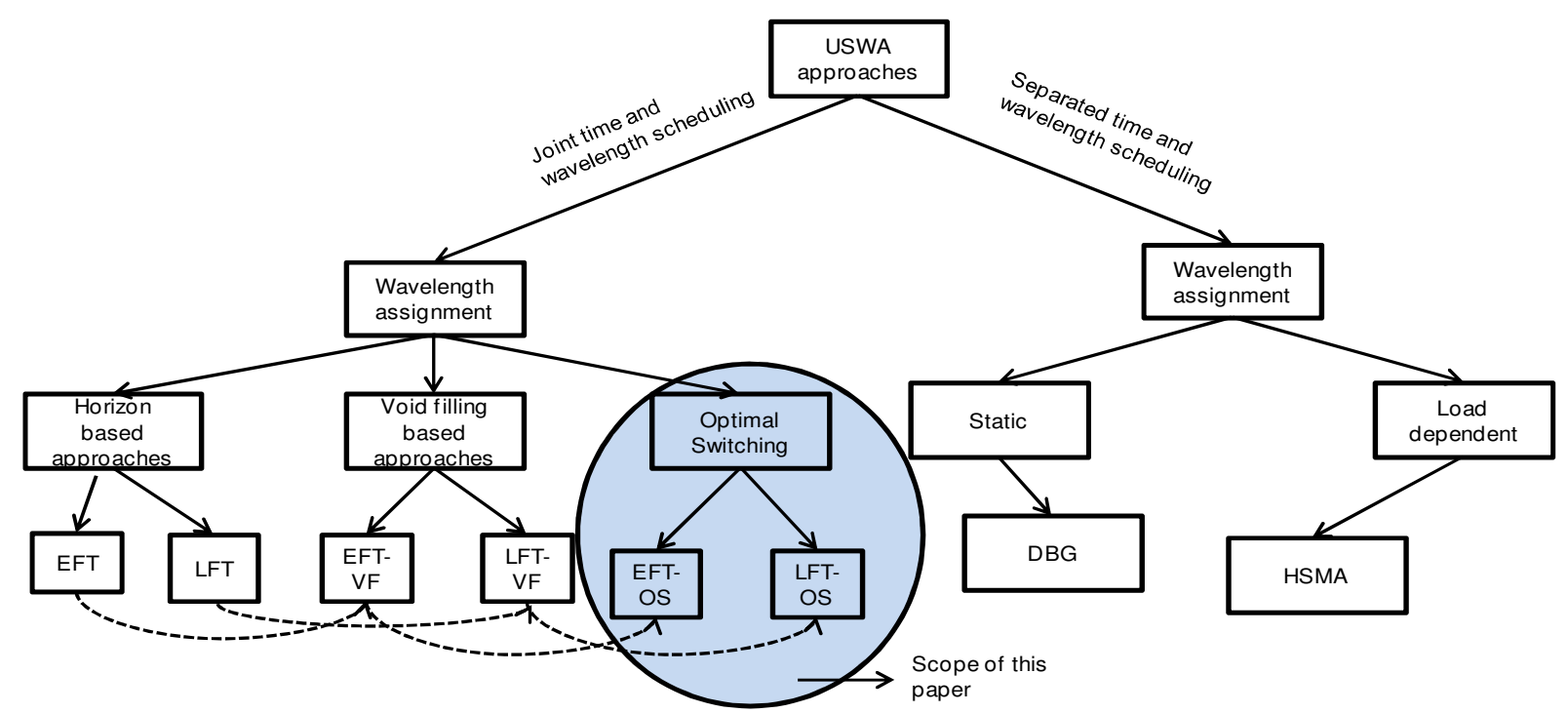

Figure 1: DBA algorithms of TWDM-PON

\section{EFT-OS ALGORITHM}

In this section, we explain the basic idea of EFT-OS. Note that the concept of optimal wavelength switching can be extended over other paradigms like LFT/EFT-VF/LFT-VF; however, we demonstrate it with EFT.

When the OLT receives a request from an ONU, there are two possibilities:

(a) In the first case, the current wavelength (the one on which the ONU is currently tuned) has the earliest finishing last transmission time and thus there is no need to switch (Fig. 2 (a)). 
(b) In the second case, any other wavelength (other than the one on which the ONU is currently tuned) has the earliest finishing last transmission time (Fig. 2(b)), and thus the OLT needs to decide whether it should switch the ONU or keep it on the same wavelength. This differentiates EFT-OS from EFT, as EFT always switches the ONU to the wavelength with the earliest finishing last transmission time.

The OLT decides to switch an ONU based on the tuning time overheads and the gain in switching. To explain it better, we introduce some symbols and notation. Let us assume that an ONU needs a tuning time of $T_{T}$ whenever switching to another wavelength, and the time gain in moving to another wavelength is $T_{G}$ (Fig. 2). First, for any possible gain, $T_{G}>T_{T}$. Secondly, since the tuning time is an overhead, it reduces the channel utilization, and thus the wavelength switching should be limited. Moreover, the penalty of overheads is more severe at a high load, and thus the wavelength switching should be avoided more at a high load than at a low load.

We discuss these issues more concretely by analytical formulations. Cycle length $L_{c}$ is composed of two components: a time $T_{u}$ for data transmission, and a time $T_{o}$ for overheads. Overheads $T_{o}$ comprise of guard bands (including control message overhead), slot underutilization and voids. Following equation can be formulated:

$$
L_{c}=T_{u}+T_{o}=\frac{\lambda L_{c}}{\mu}+T_{o}
$$

where $\lambda$ is the network traffic arrival rate, and $\mu$ is the upstream data rate. To simplify notations, $\Lambda$ denotes the normalised load $\lambda / \mu$ on a wavelength. Using these equations, $L_{c}$ is computed as:

$$
L_{c}=\frac{T_{o}}{1-\Lambda}
$$

Due to wavelength switching and an additional overhead due to tuning times $\left(T_{t}\right), T_{o}$ will be changed to $T_{o}+T_{t}$, and modified cycle time $L_{c n}$ as:

$$
L_{c n}=\frac{T_{o}+T_{t}}{1-\Lambda}
$$

Cycle length is restricted to $L_{\max }$ (for QoS considerations) and thus $\operatorname{Max}\left(T_{t}\right)$ is given as :

$$
\operatorname{Max}\left(T_{t}\right)=T_{o} \cdot \frac{L_{\max }-L_{c}}{L_{c}}
$$

Equation (4) restricts the number of switching in a cycle to:

$$
N_{s c} \leq T_{o} \cdot N_{\lambda} \cdot \frac{L_{\max }-L_{c}}{L_{c} \cdot T_{T}}
$$

where $N_{\lambda}$ is the number of wavelengths. Thus, an ONU is allowed to switch if its non-transition time (i.e. the time for which it remains on the same wavelength) is greater than No. $L_{C} / N_{S C}$, where $N_{O}$ is the number of active ONUs. For example, if $L_{\max }=2 \mathrm{~ms}, L_{c}$ is $200 \mu \mathrm{s}, T_{T}$ is $50 \mu \mathrm{s}, N_{\lambda}$ as $4, T_{o}$ as $16 \mu \mathrm{s}, N_{O}$ as 64, non-transition time of an ONU should be greater than $1.1 \mathrm{~ms}$. Note that this is a fairly long non-transition time, and it proves that wavelength switching is detrimental to the delay performance unless the tuning times are fairly short (couples of micro-seconds).

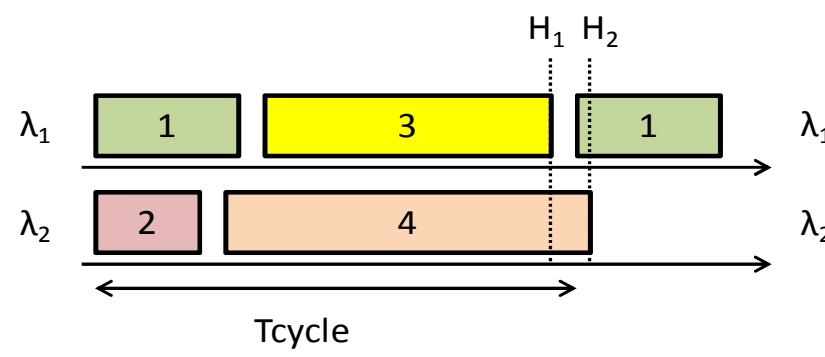

(a)

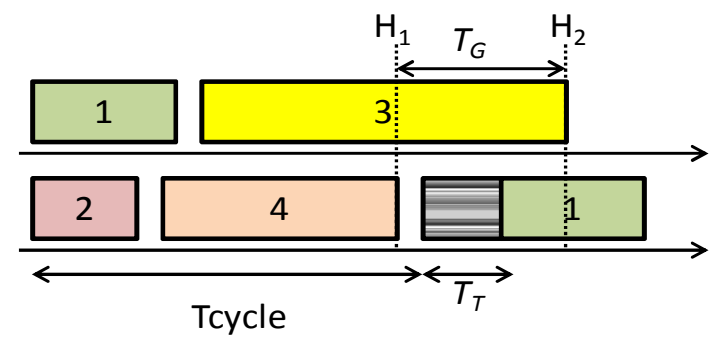

(b)

Figure 2: Conditions encountered in EFT

\section{SIMULATION RESULTS}

In OPNET, we conducted a simulation of TWDM-PON with 64 ONUs and a reach of $25 \mathrm{~km}$. We use 4 upstream wavelengths of $1 \mathrm{Gbit} / \mathrm{s}$ each and a guard band between ONUs' transmission slots of $1 \mu \mathrm{s}$. The ONUs generate self-similar traffic with a Hurst parameter of 0.8 and with a packet size varying exponentially between 64 and 1518 bytes. The granted window for an ONU is limited to a maximum of 15,500 bytes per cycle.

Figure 3 shows the delay in EFT and EFT-OS with a variation in the network load. Both EFT and EFT-OS exhibit comparable delay performance at a tuning time equal to zero. Nevertheless, for non-negligible values of the tuning time, EFT-OS performs significantly better compared to EFT. Even for a tuning time of $10 \mu \mathrm{s}$, the 
delay in EFT deteriorates quite significantly, and EFT will not be able to satisfy the quality of service requirements of different traffic classes. E.g., the delay requirement of voice traffic is within $1.5 \mathrm{~ms}$ [6], and this cannot be met, even at a low load for tuning times in the range of $10 \mu$ s or larger.

Figure 4 shows the maximum channel utilization in EFT and EFT-OS. As the tuning time increases, the channel utilization in EFT decreases largely due to the increasing penalties (overheads) associated with longer tuning times. EFT switches ONUs unaware of tuning times penalties. The channel utilization is critical only at a high network load, and EFT-OS virtually blocks switching at a high network load (Eq. 5). Thus, EFT-OS obtains a channel utilization that is high and independent of tuning times.

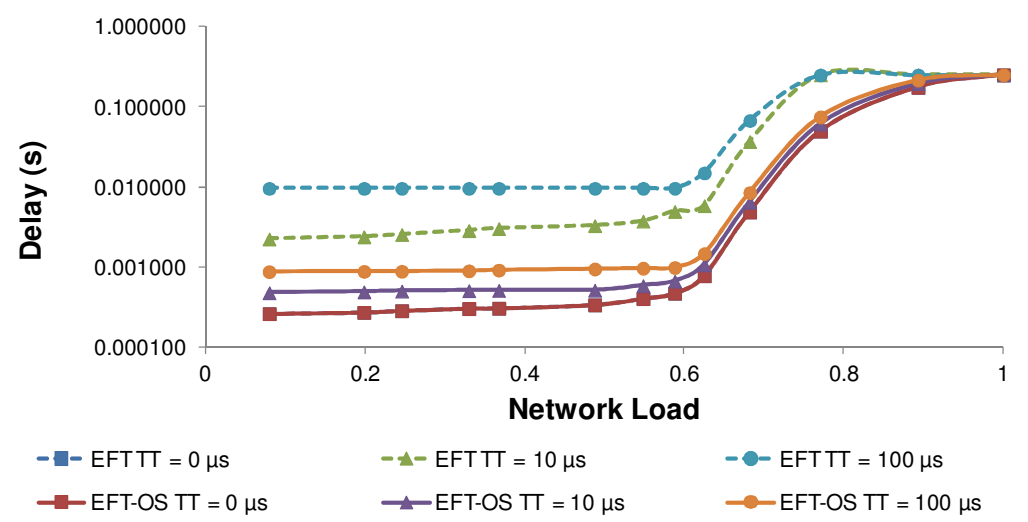

Figure 3: Average queuing delay Vs. Network load for EFT and EFT-OS with different tuning times

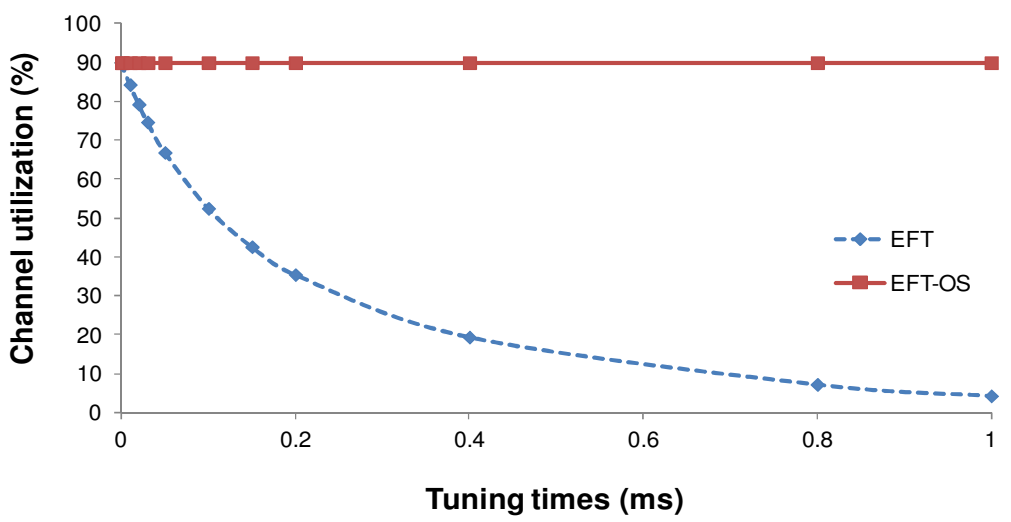

Figure 4: Maximum channel utilization for EFT and EFT-OS with a variation in laser tuning times

\section{CONCLUSION}

We proposed EFT-OS, an extension of the EFT algorithm, which adapts the frequency of wavelength switching to laser tuning times. This approach induces optimal wavelength switching, and improves delay performance and channel utilisation. While the packet delay and channel utilization in EFT degrades with an increase in the laser tuning times, EFT-OS maintains a high delay performance (delay less than $1 \mathrm{~ms}$ until a load of 0.6) and a high throughput $(90 \%)$ even with a long tuning time of $100 \mu \mathrm{s}$.

\section{REFERENCES}

[1] Michael P. McGarry , Martin Reisslein, "Bandwidth management for WDM EPONS," Journal of Optical Networking, Vol. 5, No. 9, September 2006

[2] A. Dixit, B. Lannoo, D. Colle, M. Pickavet, P. Demeester, "Novel DBA algorithm for Energy Efficiency in TWDM-PONs," submitted to ECOC 2013.

[3] K. Kanonakis and I. Tomkos, "Improving the efficiency of online upstream scheduling and wavelength assignment in hybrid WDM/TDMA EPON networks," IEEE Journal on selected areas in Communications, Vol.28, No.6, August 2010.

[4] K. Kanonakis and I. Tomkos, "Detaining online upstream scheduling in WDM/TDMA EPONs," in Proc. European Conference on Optical Communications (ECOC), Torino, Italy, Sep. 2010.

[5] K. Kanonakis and I. Tomkos, "Online upstream scheduling and wavelength assignment algorithms for WDM-EPON networks," in Proc. European Conference on Optical Communications (ECOC), Vienna, Austria, Sep. 2009.

[6] A. Dixit et al., "Dynamic Bandwidth Allocation with SLA Awareness for QoS in Ethernet Passive Optical Networks," Journal of Optical Communications and Networking, vol. 5, no. 3, March 2013. 